\title{
Retrieving microphysical properties of dust-like particles using ellipsoids: the case of refractive index
}

\author{
O. Kemppinen ${ }^{1,2}$, T. Nousiainen ${ }^{1}$, S. Merikallio ${ }^{1}$, and P. Räisänen ${ }^{1}$ \\ ${ }^{1}$ Earth Observation, Finnish Meteorological Institute, Helsinki, Finland \\ ${ }^{2}$ Department of Applied Physics, Aalto University, Espoo, Finland \\ Correspondence to: O. Kemppinen (osku.kemppinen@fmi.fi)
}

Received: 17 April 2015 - Published in Atmos. Chem. Phys. Discuss.: 19 June 2015

Revised: 11 September 2015 - Accepted: 24 September 2015 - Published: 6 October 2015

\begin{abstract}
Distributions of ellipsoids are often used to simulate the optical properties of non-ellipsoidal atmospheric particles, such as dust. In this work, the applicability of ellipsoids for retrieving the refractive index of dust-like target model particles from scattering data is investigated. This is a pure modeling study, in which stereogrammetrically retrieved model dust shapes are used as targets. The primary objective is to study whether the refractive index of these target particles can be inverted from their scattering matrices using ellipsoidal model particles. To achieve this, first scattering matrices for the target model particles with known refractive indices are computed. First, a non-negative least squares fitting is performed, individually for each scattering matrix element, for 46 differently shaped ellipsoids by using different assumed refractive indices. Then, the fitting error is evaluated to establish whether the ellipsoid ensemble best matches the target scattering matrix elements when the correct refractive index is assumed. Second, we test whether the ellipsoids best match the target data with the correct refractive index, when a predefined (uniform) shape distribution for ellipsoids is assumed, instead of optimizing the shape distribution separately for each tested refractive index. The results show not only that for both of these approaches using ellipsoids with the true refractive index produces good results but also that for each scattering matrix element even better results are acquired by using wrong refractive indices. In addition, the best agreement is obtained for different scattering matrix elements using different refractive indices. The findings imply that retrieval of refractive index of non-ellipsoidal particles whose single-scattering properties have been modeled with ellipsoids may not be reliable. Furthermore, it is demonstrated that the differences in single-scattering albedo
\end{abstract}

and asymmetry parameter between the best-match ellipsoid ensemble and the target particles may give rise to major differences in simulated aerosol radiative effects.

\section{Introduction}

Mineral dust particles are abundant constituents of the Earth's atmosphere (Zender et al., 2003). Through scattering and absorption, these particles interact with electromagnetic radiation propagating in the atmosphere, inducing effects that should be accounted for in radiative balance considerations and in atmospheric remote sensing (e.g., Durant et al., 2009; Haywood et al., 2011).

Dust particles are irregularly shaped and often inhomogeneous, making accurate computations of their singlescattering properties a challenge (Nousiainen et al., 2009; Nousiainen and Kandler, 2015; Zubko et al., 2013). These properties are needed when assessing impacts of the particles on the radiative balance or atmospheric remote sensing measurements. Single-scattering simulations in which the particle irregularity and inhomogeneity can be explicitly accounted for require, in practice, volume-integral methods such as the discrete-dipole approximation (DDA) (Draine and Flatau, 1994; Penttilä et al., 2007; Yurkin and Hoekstra, 2011). Such methods are, however, computationally demanding and become quite impractical once the particle diameter exceeds the wavelength considerably. Additionally, volumeintegral methods generally require the shape of the particles to be known accurately, which is not usually the case. Therefore, simpler methods have often been applied to compute dust particles' single-scattering properties. In particular, in 
applications such as radiative balance assessments or satellite remote sensing, where one needs to consider multiple wavelengths, these simpler but often considerably faster methods are almost exclusively used.

One such simple model geometry is that of ellipsoids. As shown by, e.g., Mishchenko et al. (1997), Dubovik et al. (2006), Bi et al. (2009), Meng et al. (2010) and Merikallio et al. (2013), a suitable distribution of ellipsoids (or their subset spheroids) can closely mimic scattering by real dust particles. To ease their application, Meng et al. (2010) offer a pre-computed database for the single-scattering properties of ellipsoids as a function of ellipsoid shape, size and refractive index. This database has been used, e.g., to model single-scattering properties of Martian dust analog particles (Merikallio et al., 2013). Regarding the ellipsoids, one fundamental question related to their use is the choice of the shape distribution. While a single ellipsoidal shape does not provide good overall fits to real dust particles' single-scattering properties, a suitable distribution of different ellipsoids may do so. The very same thing applies to spheroids: a subset of ellipsoids that consist of only rotationally symmetric ellipsoids. For spheroids, it has been shown (Merikallio et al., 2011; Nousiainen et al., 2011) that the distribution of spheroidal shapes that optimally mimics the scattering properties of dust particles does not necessarily correlate in any clear way with the physical shapes of the target particles and that at different wavelengths or for different scattering quantities the sets of spheroids that optimally reproduce scattering by a given dust sample may differ. This implies that the good performance of spheroids in reproducing scattering by real dust is in part artificial, having more to do with different spheroids forming a flexible base for fitting, rather than any close resemblance in scattering by individual dust particles and spheroids (Nousiainen et al., 2011; Lindqvist et al., 2014; Nousiainen and Kandler, 2015). In part this inconsistency is facilitated by the fact that ensembles of different non-spherical particles often have similar scattering properties (Nousiainen et al., 2012). Since generic ellipsoids are very similar model particles to spheroids, the above considerations are likely to apply also to them.

Since the third axis of ellipsoids provides an even broader base for fitting than spheroids, they are likely to be able to mimic scattering by a wide variety of different target particles. This great flexibility is, however, potentially also a great risk in remote sensing applications, as it may allow good fits to be obtained with measurements based on wrong parameters. Here, we will investigate this issue with regards to the refractive index. To this end, we will use target data comprising single and ensemble-averaged scattering matrices computed for model particles whose shapes have been derived from real, individual dust particles through stereogrammetry.

Our chosen approach is thus to use computed, synthetic data to test the inversion rather than using real measurements. This approach offers several benefits: mainly the ability to acquire the full scattering matrix at all scattering angles in ad- dition to perfect knowledge of and freedom to adjust the size, shape and composition of the target particle. Moreover, unknown measurement errors are replaced by quantifiable and somewhat controllable simulation uncertainties. Therefore, we strongly believe that a pure modeling study such as this is a highly useful approach for testing retrieval algorithms and simplified model shapes or other parameterizations.

In what follows, two types of analyses are carried out for the scattering matrix elements. First, we will seek shape distributions for ellipsoids that mimic the target data as faithfully as possible. Second, for comparison, we will perform forward modeling and adapt a pre-defined uniform shape distribution of ellipsoids. In both cases, the analyses are carried out for a variety of refractive indices. The purpose is to find out how well ellipsoids can match the target data and whether the best matches are obtained with the correct refractive index. As the refractive index is wavelength dependent, the refractive index retrieval cannot apply multiple wavelengths for additional information without assuming some kind of relationship for the refractive index at different wavelengths. Therefore, we perform the analysis only at a single wavelength. The methodology adapted and data used are presented in Sect. 2, the results are presented in Sect. 3 and, finally, conclusions are presented in Sect. 4.

\section{Methodology}

\subsection{Theoretical background}

The interaction of incident radiation with a particle can be characterized by the scattering equation. One common formulation is with the Stokes vector $[I, Q, U, V]^{\mathrm{T}}$ :

$$
\left(\begin{array}{c}
I_{\mathrm{s}} \\
Q_{\mathrm{s}} \\
U_{\mathrm{s}} \\
V_{\mathrm{s}}
\end{array}\right)=\frac{C_{\text {sca }}}{d^{2}}\left[\begin{array}{llll}
P_{11} & P_{12} & P_{13} & P_{14} \\
P_{21} & P_{22} & P_{23} & P_{24} \\
P_{31} & P_{32} & P_{33} & P_{34} \\
P_{41} & P_{42} & P_{43} & P_{44}
\end{array}\right]\left(\begin{array}{c}
I_{\mathrm{i}} \\
Q_{\mathrm{i}} \\
U_{\mathrm{i}} \\
V_{\mathrm{i}}
\end{array}\right),
$$

where subscripts " $\mathrm{i}$ " and "s" refer to incident and scattered electromagnetic wave, respectively; Stokes parameter $I$ describes the intensity, $Q$ and $U$ the linear polarization and $V$ the circular polarization of the wave; $C_{\text {sca }}$ is the scattering cross section, and $d$ the distance from the scatterer. The scattering matrix thus contains all information about a scattering event that is carried by the scattered wave.

In general, the scattering matrix has 16 elements. However, when

1. the particles are randomly oriented and

2. the particles are mirror symmetric, or particles and their mirror particles are present in equal numbers,

the scattering matrix simplifies to only six independent nonzero elements (Hovenier and van der Mee, 2000). Even when 
all these conditions are not perfectly true, ensembles of irregular particles, such as dust, seem to closely follow this simplified form of scattering matrix (Nousiainen and Kandler, 2015). Thus, in this study, we consider only the independent scattering matrix elements $P_{11}, P_{12}, P_{22}, P_{33}, P_{34}$ and $P_{44}$. Apart from $P_{11}$ and $P_{12}$, the elements can be measured in practice only in laboratory conditions, where the polarization state of the incident light can be varied. See, e.g., Muñoz et al. (2010) for an example of an apparatus for measuring the full scattering matrix. Regardless, for testing the theoretical validity of the ellipsoid retrieval, all six elements are relevant.

In this work, we consider the phase function formulation of the scattering matrix elements. The scattering matrix is thus normalized such that

$\int_{4 \pi} P_{11} d \Omega=1$.

The scattering matrix, like all dimensionless singlescattering properties, is subject to the scale invariance rule, stating that these properties depend only on the complex refractive index $m$ and the ratio of particle size and wavelength, typically denoted by the size parameter

$x=\frac{2 \pi r}{\lambda}$,

where $r$ is the characteristic radius of the particle and $\lambda$ the wavelength of the incident radiation. Here, we will take $r$ to be the radius of a volume-equivalent sphere for all the shapes considered. All considerations are done in the $(x, m)$ space, so $\lambda$ is in principle not fixed, but the parameter values considered are relevant for mineral dust particles at visible light. In this work we fix the target $m$, which can be considered to fix the analyses to a single wavelength.

\subsection{Target and ellipsoid data}

In order to evaluate the retrieval results, we need to know the actual refractive indices of our target model particles. It is also desirable that the target particles and their scattering properties are representative of real particles. One option would be to use measured scattering properties, but then the refractive index would be uncertain. We therefore choose to use synthetic data, computed using shapes derived from real dust particles by stereogrammetry. Stereogrammetry is a method for acquiring a three-dimensional structure of a particle by taking a pair of stereo images with a scanning electron microscope. The target particle is tilted between images to change the perspective. By matching known points between the images from different perspectives, the structure of one half of the particle can be determined, and a scaled mirroring technique is applied to produce the other half. The stereogrammetric method is described in detail by Lindqvist et al. (2014). It is important to note that despite being inverted from real atmospheric dust particles, the model particles used here may not be completely realistic due to inherent limitations in the stereogrammetric method. However, for the purposes of this study, it is enough that they are complex-shaped and irregular and could plausibly be close to real particles in overall shape and composition. We consider both individual stereogrammetric particles as well as an ensemble that combines their scattering matrices. The optical properties of the ensemble are derived from a simple scattering cross-section weighted average of the scattering properties for the individual particles. The particles used are described in detail by Lindqvist et al. (2014), whence we also adapt the names of the particles. We use the particles Cal (calcite), Dol (dolomite) and Agg (aggregate of several minerals, quartz being the most abundant) both individually and for the ensemble. The fourth particle, Sil (silicate, mostly chrysotile), is excluded from the ensemble because it is significantly more prolate than what was covered by the ellipsoid data set we used. However, Sil is considered individually and discussed in the text wherever its results differ from those of the other particles.

In addition to the original stereogrammetric particles, we discuss results based on their artificially roughened variations. The surfaces of the particles were modified using a Monte Carlo ray collision system that creates several small mounds and craters at the surface, therefore reducing the artificial surface smoothness caused by the stereogrammetric method while keeping the overall particle shapes and volumes nearly intact. The roughening method used is described in more detail by Kemppinen et al. (2015).

In principle, the roughened particles may represent the real physical targets of the stereogrammetry study more than the original stereogrammetric shapes due to the fact that the stereogrammetry method can not recreate the fine surface roughness of the physical particles. However, the roughening is based on arbitrarily chosen parameters that have not been related in any way with the (possible) roughness characteristics of the target shapes considered here, or any other dust particles. Therefore, we consider the original unroughened particles as the primary target and use the roughened versions primarily to study the sensitivity of the results to particles' surface roughness. In particular, if moderate changes in surface roughness significantly alter the results of the refractive index retrieval, it can be said that the retrieval algorithm is too sensitive, or the impact of roughness on scattering dominates that of the refractive index.

For the scattering calculations we used version 1.2 MPI of ADDA (Yurkin and Hoekstra, 2011), which implements the discrete-dipole approximation (Purcell and Pennypacker, 1973). DDA allows light scattering simulations by an arbitrarily shaped collection of dipoles, which enables great flexibility in representing irregular shapes. DDA is generally accurate as long as the target dipole resolution is sufficient. In this work, the target shapes for all size parameters were discretized into roughly 800000 dipoles. The value $y=|m| k l$, where $m$ is the refractive index, $k$ is the wave number and $l$ 
is the dipole size, is typically used to evaluate the applicability of the DDA method. The largest $y$ value for the particles in this study was less than 0.6, which is below the commonly cited DDA accuracy limit of $y \leq 1$ (Zubko et al., 2010). Moreover, we performed tests with spheroidal shapes, using the sane number of dipoles as for the stereogrammetric shapes, and compared the results explicitly to reference spheroid calculations made with the T-matrix method (Waterman, 1965; Mishchenko and Travis, 1998). The DDA simulations match very closely with the T-matrix simulations (not shown), and although it does not prove DDA accuracy for more complex particles, it is nevertheless a positive sign.

The scattering of each target shape was averaged over 8192 random orientations for all size parameters. Figure 1 shows computer-generated renderings of the DDA representations of the target particles. The images were generated with only one-eighth of the dipole resolution compared to the scattering simulations.

ADDA was run on the Finnish Meteorological Institute Cray XC30 supercomputer Voima, using 64 computer cores per simulation. Additionally, 10 concurrent simulations were run in parallel to reduce the total run time. With this setup, the total amount of CPU time used was approximately 46 $000 \mathrm{~h}$. The resulting scattering matrix elements of the target particles are shown in Fig. 2. There are clear differences between the values and angular dependencies of the scattering matrix elements of different particles, as is expected given their different shapes.

The scattering matrices for ellipsoids are taken from the database by Meng et al. (2010), where the optical characteristics are tabulated for size parameters of the largest dimension ranging from 0.025 up to 1000 . This gives values larger or equal (for the sphere) to the volume-equivalent size parameter used in this study. The database also provides the corresponding volume-equivalent size parameters, which are used here. The database includes the six independent scattering matrix elements and other optical characteristics, such as the scattering cross section, which we use in the integration of the ensemble properties. The range of refractive indices includes, in principle, the real part range from 1.10 up to 2.10 and the imaginary part from 0.0005 to 0.5 . In practice, not all real and imaginary part combinations have been computed. Here, we will consider only ellipsoids with real parts of the refractive ranging from 1.4 to 1.6 and imaginary parts from 0.0005 to 0.02 , for which all possible combinations are available. In total, 46 different ellipsoids with axis ratios ranging from 1.0 to 3.3 are considered, including a sphere, but excluding some nearly spherical ellipsoids, as was done by Merikallio et al. (2013), to reduce the number of shapes to be considered and to facilitate the fitting.

The particle size distribution for both the ellipsoid and the target data is a lognormal distribution with $\sigma_{\mathrm{g}}=2.0$ and $r_{\mathrm{g}}=0.4 \mu \mathrm{m}$ and a cutoff at size parameter 20. All calculations were done with a size parameter resolution of 0.5 for the sizes $0.5 \ldots 10$ and a size parameter resolution of 1.0 for the sizes $11 \ldots 20$.

\subsection{Fitting and error definitions}

We investigate how well scattering by ellipsoid ensembles can match scattering matrix elements of target particles. Specifically, the scattering matrix elements of the individual ellipsoids form a basis, and we seek the linear combination of the shapes that minimizes the squared difference to the target data. We want the weights of the individual particles in the ensemble to have properties of probabilities, and that imposes two requirements, described by Eqs. (4) and (5):

$\forall l, p_{l} \in[0,1]$,

and

$\sum_{l} p_{l}=1$

where the individual shapes are denoted by $\xi_{l}$ and the corresponding weights are denoted by $p_{l}$.

Due to the requirements in Eqs. (4) and (5), we cannot use a standard least-squares fitting algorithm to minimize the error functions. Instead, we adopt a non-negative least squares algorithm from Kahnert et al. (2002), recently used by Merikallio et al. (2011) and Nousiainen et al. (2011), and described below.

The best-fit ensemble will be

$P_{i j}^{\mathrm{fit}}(\theta)=\sum_{l} p_{l} P_{i j}\left(\theta ; \xi_{l}\right)$

where $P_{i j}\left(\theta ; \xi_{l}\right)$ are the scattering matrix elements of the shape $\xi_{l}$, and $l \in\{0,1,2, \ldots, L-1\}$, where $L$ is the total number of different ellipsoid shapes.

Instead of fitting $p_{l}$ directly, we initially fit parameters $q_{l}$. To determine $q_{l}$ for $P_{11}$, the quantity to be minimized is

$e_{11}=\sum_{\theta=0^{\circ}}^{180^{\circ}}\left[\frac{P_{11}^{\mathrm{ref}}(\theta)-\sum_{l} q_{l}^{2} P_{11}\left(\theta ; \xi_{l}\right)}{P_{11}^{\mathrm{ref}}(\theta)}\right]^{2}$,

where $P_{11}^{\text {ref }}(\theta)$ is the $P_{11}$ element of the target scatterer. This form is designed to eliminate the strong weighing of forwardscattering angles in the fitting due to the typically very high values of $P_{11}$ at those angles compared to larger scattering angles. We use an uniform $\theta$ grid with a step size of $0.5^{\circ}$.

For $P_{i j}, i j \in\{12,22,33,34,44\}$, we minimize

$e_{i j}=\sum_{\theta=0^{\circ}}^{180^{\circ}}\left[\frac{P_{i j}^{\mathrm{ref}}(\theta)}{P_{11}^{\mathrm{ref}}(\theta)}-\sum_{l} \frac{q_{l}^{2} P_{i j}\left(\theta ; \xi_{l}\right)}{P_{11}\left(\theta ; \xi_{l}\right)}\right]^{2}$,

where $P_{i j}^{\text {ref }}(\theta)$ is the corresponding $P_{i j}$ element of the target scatterer. The $\theta$ grid is the same as above. The minimization is done with Levenberg-Marquardt method, using a uniform 


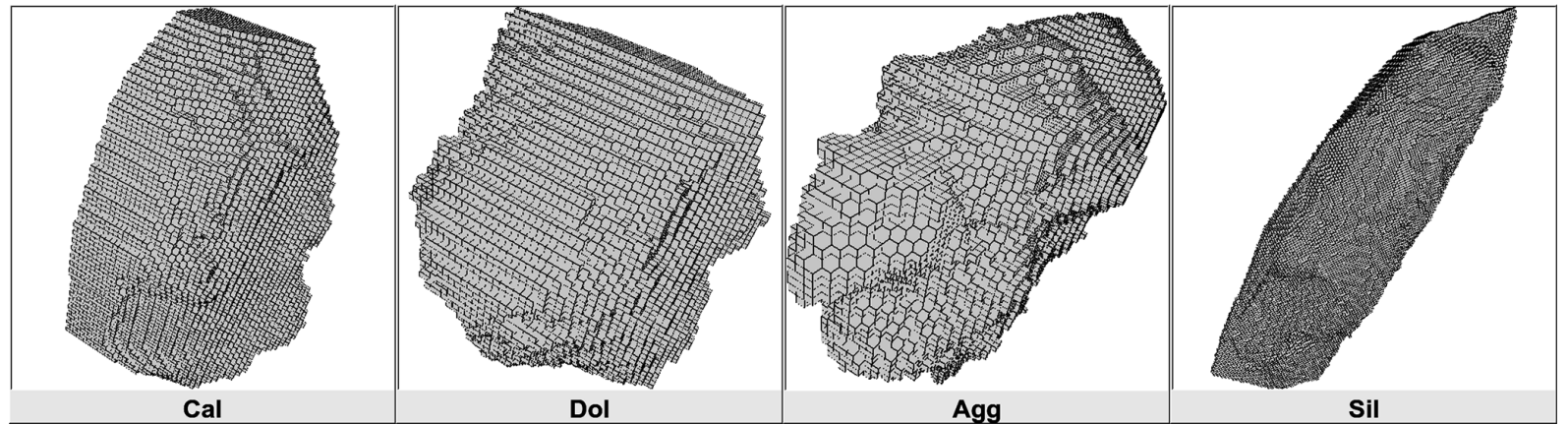

Figure 1. Renders of the DDA representations of the four target particles. The particles are depicted here with one-eighth of the dipole resolution compared to the calculations.
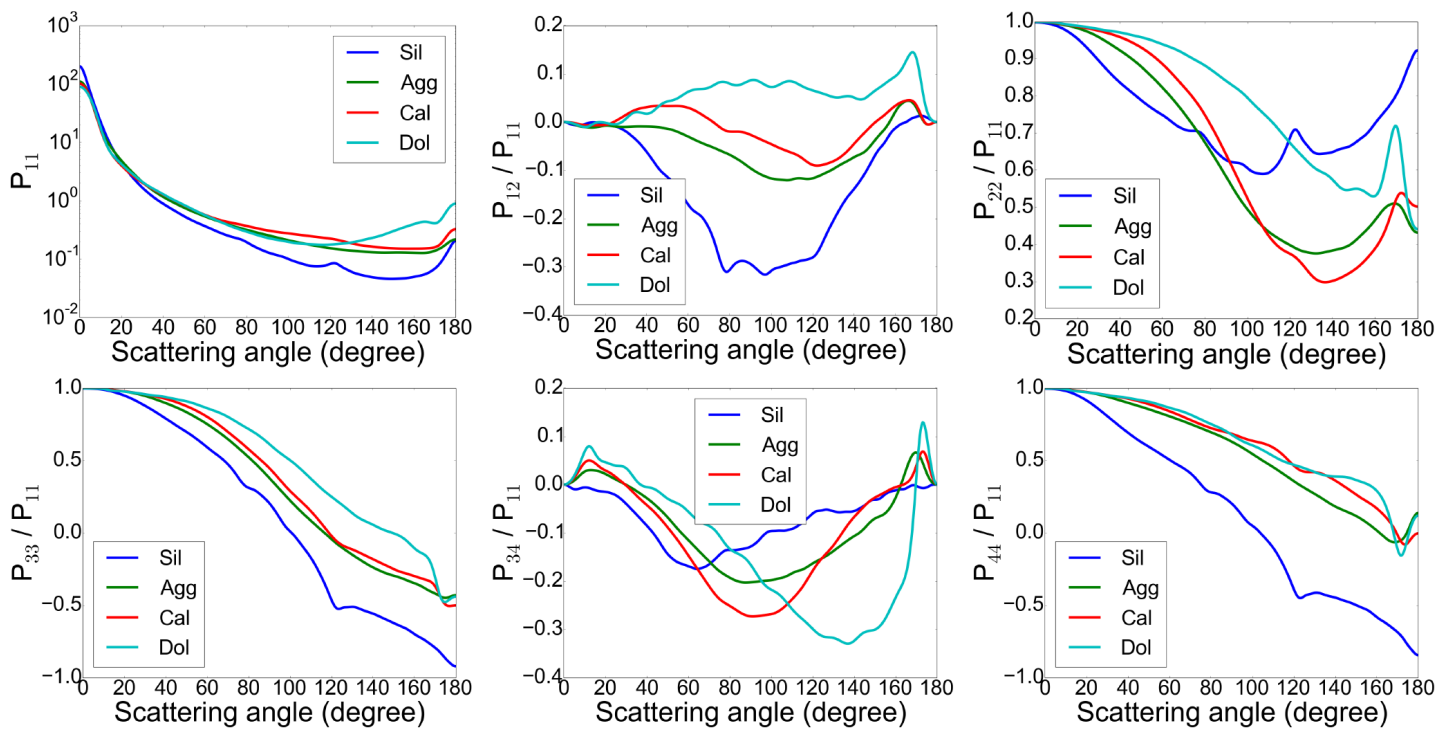

Figure 2. The six independent non-zero scattering matrix elements of the target particles integrated over the size distribution.

initial shape distribution (Press et al., 1992). Due to the fact that the Levenberg-Marquardt method is not guaranteed to find the global optimum, we performed tests with random initial shape distributions. In total, 100 additional shape distributions were tested for $P_{11}$. Of these, 94 tests resulted in the same optimal shape distribution as the uniform one, whereas 6 resulted in a significantly larger fit error. Each of these six had a very exotic initial distribution, such as only one shape being present, in which case the algorithm might not be able to converge accurately. We conclude that the uniform initial shape distribution is likely to produce a good and consistent optimum. Theoretically, it is possible that there are better optima than those found by this method, but finding them would likely require some a priori information, which would very probably not be available to an instrument team doing retrieval by fitting.
The final normalization for $p_{l}$ is done by

$p_{l}=\frac{q_{l}^{2}}{\sum_{l} q_{l}^{2}}$.

This ensures that the weights are positive and properly normalized.

In Sect. 3 we show scattering matrix element errors $E_{i j}$ (briefly, "scattering errors"), which are based on $e_{i j}$ from Eqs. (7) and (8):

$E_{i j}=\sqrt{e_{i j}}$,

where the square root is utilized to enhance detail in the visualizations near the minimum of $e_{i j}$, which is the most interesting region in retrieval applications, where the minimum error is typically sought. 
Table 1. Number of ellipsoids employed in the fits, and the scattering errors. The numbers shown are means across all 40 refractive indices and four individual particles plus the three-particle ensemble. An ellipsoid is counted as part of the ensemble when its relative weight is at least $0.1 \%$.

\begin{tabular}{lcc}
\hline Element & $\begin{array}{c}\text { No. of ell. in the ensemble, } \\
\text { non-roughened ref. particle }\end{array}$ & $\begin{array}{c}\text { No. of ell. in the ensemble, } \\
\text { roughened ref. particle }\end{array}$ \\
\hline$P_{11}^{\text {fit }}$ & 4.57 & 4.72 \\
$P_{12}^{\text {fit }}$ & 8.43 & 8.15 \\
$P_{22}^{\text {fit }}$ & 4.67 & 3.58 \\
$P_{33}^{\text {fit }}$ & 6.32 & 5.29 \\
$P_{34}^{\text {fit }}$ & 8.41 & 8.06 \\
$P_{44}^{\text {fit }}$ & 3.58 & 2.87 \\
\hline$E_{\text {Element }}$ & $E_{i j}$, & $E_{i j}$, \\
& non-roughend ref. particle & roughened ref. particle \\
\hline$E_{11}$ & 1.9498 & 1.6635 \\
$E_{12}$ & 0.0029 & 0.0084 \\
$E_{22}$ & 0.0783 & 0.2250 \\
$E_{33}$ & 0.0647 & 0.3377 \\
$E_{34}$ & 0.0104 & 0.0588 \\
$E_{44}$ & 0.2550 & 1.4398 \\
\hline
\end{tabular}

In the case of a fixed, uniform shape distribution, instead of using $p_{l}$ from the least squares solution, we use

$p_{l}=\frac{1}{L}$

in the $E_{i j}$ calculations to get an equal weight for each shape while satisfying Eqs. (4) and (5). The $E_{i j}$ calculations for the fixed shape distribution are otherwise identical.

\section{Results}

We investigate the validity of the ellipsoid ensemble assumption by fitting scattering matrix elements of a set of ellipsoids to those of target particles, as described in Sect. 2.3. The fitting is done separately for ellipsoids with 40 different refractive indices, $m_{\mathrm{r}}+i m_{\mathrm{i}}$, where $m_{\mathrm{r}} \in$ $\{1.40,1.45,1.50,1.55,1.60\}$ and $m_{\mathrm{i}} \in\{0.0005,0.001,0.002$, $0.004,0.005,0.008,0.01,0.02\}$. The number of ellipsoids that the fitting algorithm employs in the best fit of each element is shown in Table 1. We define an ellipsoid to be employed if its weight is $0.1 \%$ or larger in the ensemble. The numbers displayed in Table 1 are means across all refractive indices and all the individual particles in addition to the particle ensemble. The results for original and roughened versions of the target particles are shown separately. First, we see that only a relatively small number of the 46 different ellipsoids is used at any time. Second, we see that some elements require significantly more ellipsoids for the best fit than others. Third, we see that the roughened particles, with their reduced shape regularity, require a slightly, but systematically, smaller number of ellipsoids for the optimal fit.
Additionally, Table 1 shows the mean scattering error for the elements, also averaged over all the individual particles and the ensemble and across all refractive indices. The particles with the added surface roughness seem to produce worse optimum fits on average, though in some individual cases the error is smaller. Furthermore, different elements produce vastly different scattering errors. It seems that there is a modest anticorrelation between the number of ellipsoids in the optimum fit and the mean error of the fit. For example, $P_{11}$ and $P_{44}$ are the elements with the smallest number of ellipsoids used, and they are the two elements with the largest mean fit errors. Correspondingly, $P_{12}$ and $P_{34}$ are the two elements with the highest number of ellipsoids, and their mean fitting errors are the smallest.

Figures 3 and 4 in Sect. 3.1 and 3.2 show the contour plots of the scattering error of the minimum error ellipsoid shape distributions as a function of the real and imaginary parts of the refractive index. The grids in the plots are linearly interpolated to provide a better overview of the data. In the plots, the true real and imaginary refractive indices are shown at the intersection of black lines at $1.5+i 0.004$, called the true refractive index $\left(m_{\text {true }}\right)$ below. The refractive index with the minimum error of all the ellipsoid refractive indices is marked with a white circle and is called the optimum refractive index $\left(m_{\mathrm{opt}}\right)$ in the text. The key question to be investigated is what the relationship between $m_{\text {true }}$ and $m_{\text {opt }}$ is, specifically whether they are close enough for the retrieval process to be considered valid. For this, we have added extra relative error contour lines in the figures. A solid white contour line shows relative errors that are up to $150 \%$ of the minimum error, and a dashed white contour line shows relative errors that are up to $200 \%$ of the minimum error. The values with less than $200 \%$ of the relative error are referred in the text as good fits for the sake of simplicity of language. It should be noted that the choice of $200 \%$ is arbitrary, and for some of the values below this error threshold the retrieved refractive index can nevertheless deviate substantially from the true refractive index.

We show the scattering errors only for the ensemble of the three original unroughened stereogrammetric particles Cal, Agg and Dol. Sil was excluded from the ensemble because of its extreme axis ratio, which was not covered by the ellipsoids used in the study. The figures for individual particles are not shown, because in most cases the plots for the individual particles match those of the ensemble relatively well. If there are discrepancies, they are noted in the text. Similarly, the results of the roughened particles are not shown but are described in the text whenever noteworthy.

The results for individual particles are shown in Tables 2 and 3. The numbers in these tables are the complex refractive index differences $\Delta m=m_{\mathrm{opt}}-m_{\text {true }}$, and they describe whether the optimum refractive index parts are larger or smaller than the true refractive index. Values in bold indicate that $m_{\mathrm{opt}}$ is at the edge of the computational domain, mean- 


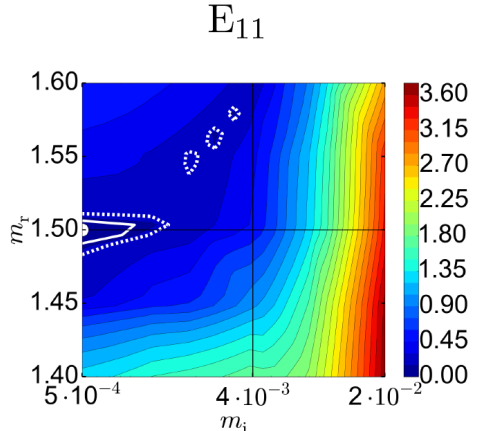

$\mathrm{E}_{33}$

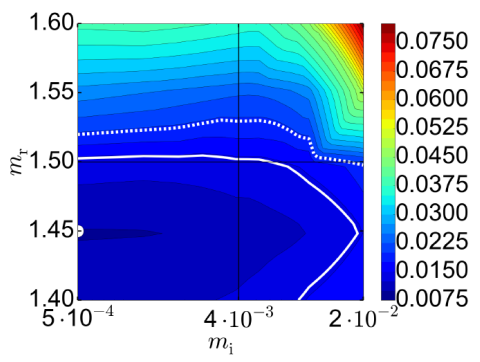

$\mathrm{E}_{12}$

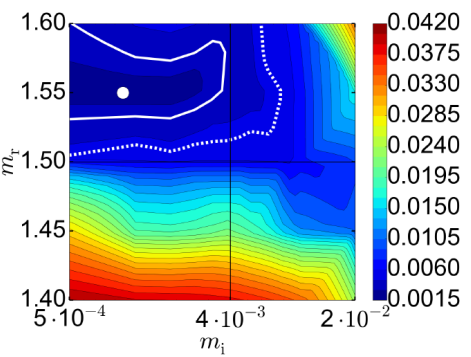

$\mathrm{E}_{34}$

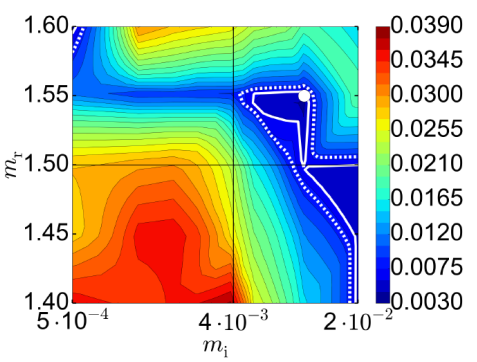

$\mathrm{E}_{22}$

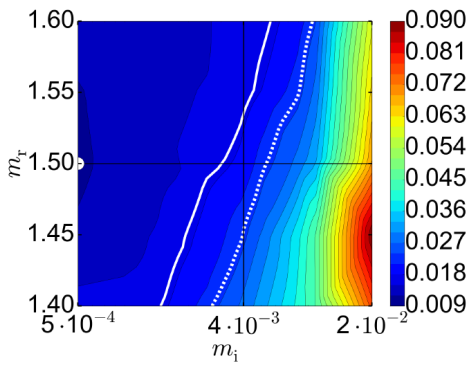

$\mathrm{E}_{44}$

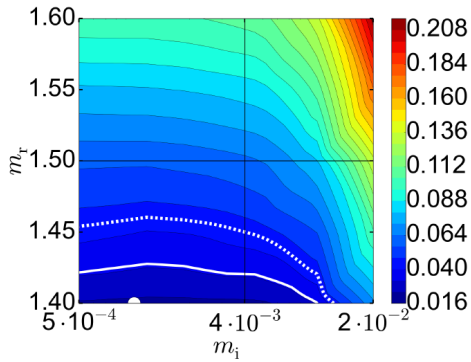

Figure 3. Scattering error $E_{i j}$ for different scattering matrix elements for the particle ensemble of Cal, Dol and Agg, when the ellipsoid shape weights are treated as free parameters to be fitted. Shading corresponds to different absolute errors, as described by the color bar, whereas white contour lines describe relative errors that are $150 \%$ (inner solid line) and $200 \%$ (outer dashed line) of the minimum error. The white circle marks the refractive index for which the minimum error was found, and the black horizontal and vertical lines mark the reference real and imaginary refractive index.
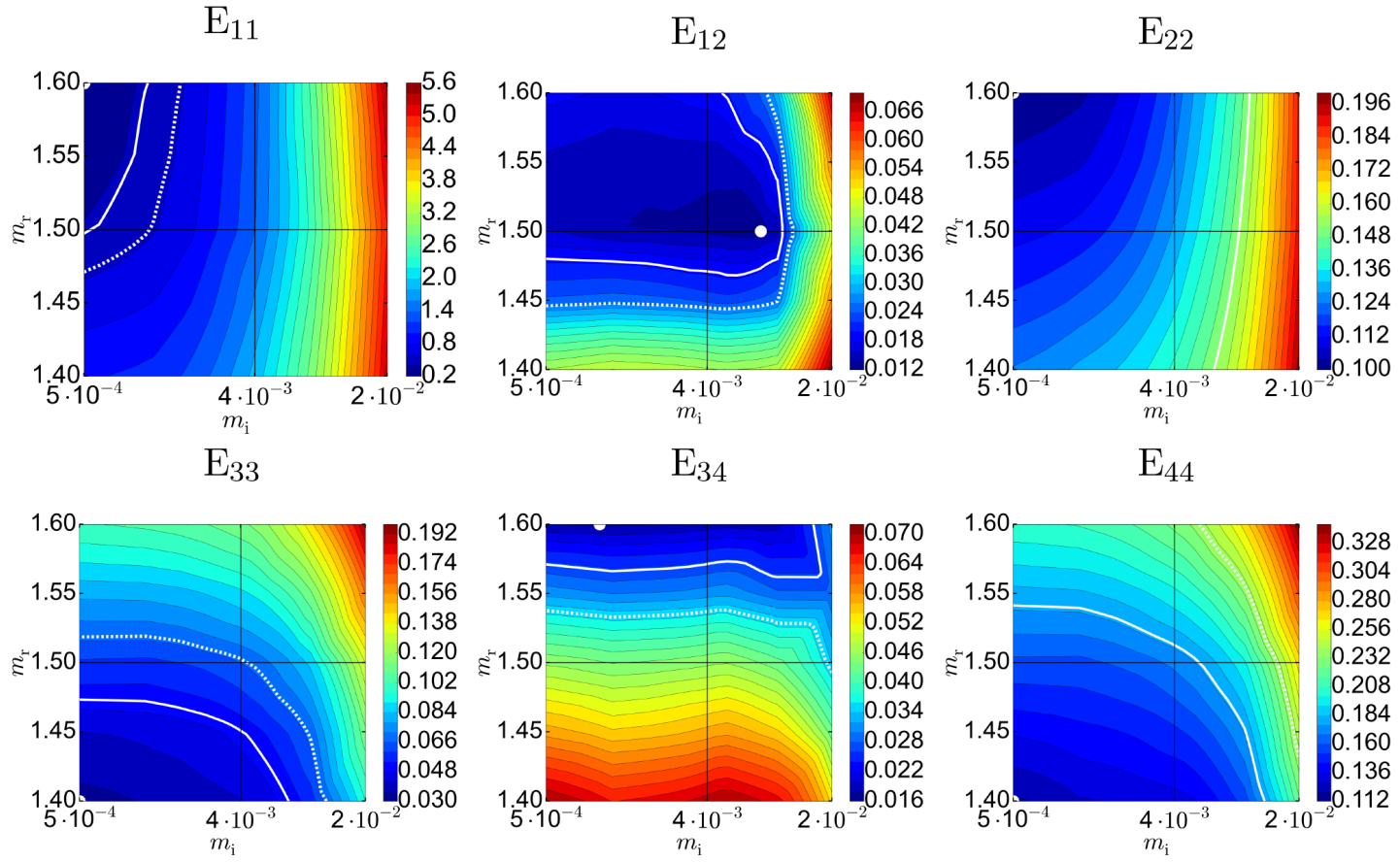

Figure 4. Scattering error $E_{i j}$, similarly to Fig. 3, for different scattering matrix elements for the particle ensemble of Cal, Dol and Agg, when the ellipsoid shape distribution is fixed to be uniform, with equal weights for each shape. 
Table 2. Complex refractive index errors for the fitted shape distribution. The values in bold correspond to retrieved refractive indices at the boundary of our refractive index domain and therefore could be even more erroneous in reality.

\begin{tabular}{lrrrrrr}
\hline $\begin{array}{l}\text { Original particles } \\
\text { Particle }\end{array}$ & $P_{11}$ & $P_{12} / P_{11}$ & $P_{22} / P_{11}$ & $P_{33} / P_{11}$ & $P_{34} / P_{11}$ & $P_{44} / P_{11}$ \\
\hline Ensemble & $0.00-i \mathbf{0 . 0 0 3 5}$ & $0.05-i 0.0030$ & $0.00-i \mathbf{0 . 0 0 3 5}$ & $-0.05-i \mathbf{0 . 0 0 3 5}$ & $0.05+i 0.0060$ & $-\mathbf{0 . 1 0}-i 0.0030$ \\
Cal & $0.05-i \mathbf{0 . 0 0 3 5}$ & $-0.05+i 0.0060$ & $\mathbf{0 . 1 0}-i \mathbf{0 . 0 0 3 5}$ & $-0.05-i \mathbf{0 . 0 0 3 5}$ & $-\mathbf{0 . 1 0}-i \mathbf{0 . 0 0 3 5}$ & $-\mathbf{0 . 1 0}-i 0.0030$ \\
Dol & $\mathbf{0 . 1 0}+i 0.0010$ & $0.05+i 0.0010$ & $-0.05+i 0.0010$ & $-\mathbf{0 . 1 0}+i 0.0010$ & $0.05+i 0.0060$ & $-\mathbf{0 . 1 0}-i 0.0030$ \\
Agg & $0.05-i \mathbf{0 . 0 0 3 5}$ & $0.00-i 0.0030$ & $\mathbf{0 . 1 0}-i \mathbf{0 . 0 0 3 5}$ & $\mathbf{- 0 . 1 0}-i \mathbf{0 . 0 0 3 5}$ & $0.05+i \mathbf{0 . 0 1 6 0}$ & $-\mathbf{0 . 1 0}-i \mathbf{0 . 0 0 3 5}$ \\
Sil & $\mathbf{0 . 1 0}+i \mathbf{0 . 0 1 6 0}$ & $0.00+i \mathbf{0 . 0 1 6 0}$ & $\mathbf{0 . 1 0}-i 0.0030$ & $\mathbf{0 . 1 0}+i 0.0010$ & $0.00-i \mathbf{0 . 0 0 3 5}$ & $\mathbf{0 . 1 0}+i \mathbf{0 . 0 1 6 0}$ \\
\hline Roughened particles & & & & & \\
Particle & $P_{11}$ & $P_{12} / P_{11}$ & $P_{22} / P_{11}$ & $P_{33} / P_{11}$ & $P_{34} / P_{11}$ \\
\hline Ensemble & $0.05-i 0.0020$ & $0.05+i 0.0010$ & $\mathbf{0 . 1 0}-i \mathbf{0 . 0 0 3 5}$ & $\mathbf{- 0 . 1 0}-i \mathbf{0 . 0 0 3 5}$ & $\mathbf{0 . 1 0}+i 0.0060$ & $-\mathbf{0 . 1 0}-i \mathbf{0 . 0 0 3 5}$ \\
Cal & $0.05-i \mathbf{0 . 0 0 3 5}$ & $-0.05+i 0.0060$ & $\mathbf{0 . 1 0}-i \mathbf{0 . 0 0 3 5}$ & $\mathbf{- 0 . 1 0}-i \mathbf{0 . 0 0 3 5}$ & $\mathbf{0 . 1 0}+i 0.0060$ & $-\mathbf{0 . 1 0}-i \mathbf{0 . 0 0 3 5}$ \\
Dol & $\mathbf{0 . 1 0}+i 0.0010$ & $\mathbf{0 . 1 0}-i 0.0020$ & $\mathbf{- 0 . 1 0}-i \mathbf{0 . 0 0 3 5}$ & $\mathbf{- 0 . 1 0}+i 0.0010$ & $\mathbf{0 . 1 0}+i \mathbf{0 . 0 1 6 0}$ & $-\mathbf{0 . 1 0}-i \mathbf{0 . 0 0 3 5}$ \\
Agg & $0.05-i \mathbf{0 . 0 0 3 5}$ & $0.05+i 0.0010$ & $\mathbf{0 . 1 0}-i \mathbf{0 . 0 0 3 5}$ & $\mathbf{- 0 . 1 0}-i \mathbf{0 . 0 0 3 5}$ & $\mathbf{0 . 1 0}+i \mathbf{0 . 0 1 6 0}$ & $-\mathbf{0 . 1 0}-i \mathbf{0 . 0 0 3 5}$ \\
Sil & $\mathbf{0 . 1 0}+i \mathbf{0 . 0 1 6 0}$ & $-\mathbf{0 . 1 0}-i 0.0020$ & $\mathbf{0 . 1 0}-i 0.0030$ & $\mathbf{0 . 1 0}+i 0.0040$ & $0.00-i \mathbf{0 . 0 0 3 5}$ & $\mathbf{0 . 1 0}+i 0.0060$ \\
\hline
\end{tabular}

ing that possibly even better fits might have been possible with an extended refractive index range.

It is important to note that the similarities in the $m_{\mathrm{opt}}$ values and error contours between different particles are not due to the scattering matrix elements of these particles being very similar. As seen in Fig. 2, there are considerable differences in the scattering matrix elements between the particles.

\subsection{Fitted shape distribution}

Figure 3 shows the results based on fitting the ellipsoid ensemble to the scattering matrix elements of the target scatterer. The good-fit region of $P_{11}$ is located on the small imaginary refractive index side, trending to larger real refractive indices. The optimum refractive index is found at $1.5+$ $i 0.0005$. Cal and Agg follow the ensemble result closely, but Dol is slightly different in that the good-fit region reaches even the minimum real refractive indices. Sil behaves differently: only a relatively local subspace of refractive indices produces decent fits. This region is located at the maximum real and imaginary refractive index, where the fit for the other particles is very poor.

The good-fit band for $P_{12} / P_{11}$ of the ensemble covers most of the refractive index space of $m_{\mathrm{r}}$ greater than 1.5 apart from a local, but prominent, local maximum at the top-right corner, where both the real and imaginary parts of the refractive index have their largest values. Low real refractive indices produce poor fits, in particular if the imaginary part is small as well. All the constituents of the ensemble behave very similarly to the ensemble. The error map for Sil, however, is almost an inverse of the error map of the ensemble. That is, the good-fit refractive indices of the ensemble produce poor fits for Sil and vice versa.

$P_{22} / P_{11}$ for the ensemble has the optimum refractive index at $1.5+i 0.0005$, but all refractive indices with low-tomedium imaginary part, regardless of the real part, seem to provide good fits. Large imaginary refractive indices, espe- cially if the real part is small, provide poor fits. Cal and Agg are very close to the ensemble, but Dol and Sil differ. For Sil, the imaginary refractive index dependence is similar in that large $m_{\mathrm{i}}$ provide poor fits, but the difference is that low real parts provide poor fits as well. For Dol, compared to the ensemble, there is an additional error maximum at high real and low imaginary refractive indices, and only low real refractive indices and low-to-medium imaginary refractive indices provide good fits.

For $P_{33} / P_{11}$, all real refractive indices below 1.5 provide reasonably good fits for the ensemble, especially if the imaginary part is small as well. Refractive indices with a large real part and a large imaginary part provide very poor fits. This behavior is true for Cal, Dol and Agg, but Sil behaves differently. For Sil, the optimum refractive index is at $1.6+i 0.004$, and only refractive indices very near that point provide decent fits. In particular, low real parts, which are good for the other particles and the ensemble, provide very poor fits for Sil.

Compared to the previous elements, the behavior of $P_{34} / P_{11}$ for the ensemble is less straightforward in terms of the refractive indices. There are narrow bands along both axes where the errors are small, but even small changes in the refractive index might have a very large effect on the magnitude of the error. This is in contrast to the elements analyzed above, where the gradients were often relatively mild on both of the refractive index axes. The worst fits are found at low real and low imaginary refractive indices. Dol and Agg follow the behavior of the ensemble relatively well, but $\mathrm{Cal}$ and Sil differ. For Cal, the difference is that very small real refractive indices produce decent fits, as do very small imaginary refractive indices, while values around $m=1.45+i 0.002$ are still having high errors. For Sil the good-fit region is centered at $m=1.5+i 0.0005$, expanding to large imaginary refractive indices but staying localized at the real axis. 
Table 3. Complex refractive index errors for the uniform shape distribution.

\begin{tabular}{|c|c|c|c|c|c|c|}
\hline $\begin{array}{l}\text { Original pa } \\
\text { Particle }\end{array}$ & $P_{11}$ & $P_{12} / P_{11}$ & $P_{22} / P_{11}$ & $P_{33} / P_{11}$ & $P_{34} / P_{11}$ & $P_{44} / P_{11}$ \\
\hline Ensemble & $0.10-i 0.0035$ & $0.00+i 0.0040$ & $0.10-i 0.0035$ & $-\mathbf{0 . 1 0}-i \mathbf{0 . 0 0 3 5}$ & $\mathbf{0 . 1 0}-i 0.0030$ & $-0.10-i \mathbf{0 . 0 0 3 5}$ \\
\hline Cal & $0.10-i 0.0035$ & $-0.05+i 0.0010$ & $\mathbf{0 . 1 0}-i \mathbf{0 . 0 0 3 5}$ & $-0.05-i \mathbf{0 . 0 0 3 5}$ & $0.10-i 0.0035$ & $-0.10-i \mathbf{0 . 0 0 3 5}$ \\
\hline Dol & $0.10-i 0.0035$ & $\mathbf{0 . 1 0}-i \mathbf{0 . 0 0 3 5}$ & $-0.10-i 0.0035$ & $-0.10-i \mathbf{0 . 0 0 3 5}$ & $-0.10+i 0.0160$ & $-0.10-i 0.0035$ \\
\hline Agg & $0.00-i 0.0020$ & $-0.05+i \mathbf{0 . 0 1 6 0}$ & $\mathbf{0 . 1 0}-i \mathbf{0 . 0 0 3 5}$ & $0.00+i 0.0000$ & $0.10+i 0.0160$ & $-0.10-i 0.0035$ \\
\hline Sil & $\mathbf{0 . 1 0}+i \mathbf{0 . 0 1 6 0}$ & $-0.10+i 0.0160$ & $\mathbf{0 . 1 0}+i 0.0000$ & $0.10+i \mathbf{0 . 0 1 6 0}$ & $\mathbf{0 . 1 0}+i \mathbf{0 . 0 1 6 0}$ & $\mathbf{0 . 1 0}+i \mathbf{0 . 0 1 6 0}$ \\
\hline \multicolumn{7}{|c|}{ Roughened particles } \\
\hline Particle & $P_{11}$ & $P_{12} / P_{11}$ & $P_{22} / P_{11}$ & $P_{33} / P_{11}$ & $P_{34} / P_{11}$ & $P_{44} / P_{11}$ \\
\hline Ensemble & $\mathbf{0 . 1 0}-i 0.0020$ & $0.05+i 0.0010$ & $0.10-i 0.0035$ & $-0.10-i 0.0035$ & $0.10+i 0.0160$ & $-0.10-i 0.0035$ \\
\hline Cal & $\mathbf{0 . 1 0}-i 0.0020$ & $-0.05+i 0.0010$ & $0.10-i 0.0035$ & $-\mathbf{0 . 1 0}-i \mathbf{0 . 0 0 3 5}$ & $0.10+i 0.0160$ & $-0.10-i 0.0035$ \\
\hline Dol & $0.10-i \mathbf{0 . 0 0 3 5}$ & $\mathbf{0 . 1 0}-i \mathbf{0 . 0 0 3 5}$ & $-0.10-i 0.0035$ & $-0.10-i 0.0035$ & $0.10+i \mathbf{0 . 0 1 6 0}$ & $-0.10-i 0.0035$ \\
\hline Agg & $0.00+i 0.0000$ & $-0.05+i \mathbf{0 . 0 1 6 0}$ & $\mathbf{0 . 1 0}-i \mathbf{0 . 0 0 3 5}$ & $-0.05-i \mathbf{0 . 0 0 3 5}$ & $0.10+i 0.0160$ & $-0.10-i 0.0035$ \\
\hline Sil & $0.10+i 0.0160$ & $-0.10+i 0.0160$ & $0.10-i 0.0035$ & $\mathbf{0 . 1 0}+i \mathbf{0 . 0 1 6 0}$ & $0.10+i 0.0160$ & $\mathbf{0 . 1 0}+i \mathbf{0 . 0 1 6 0}$ \\
\hline
\end{tabular}

$P_{44} / P_{11}$ is nearly identical to $P_{33} / P_{11}$ for the ensemble and all individual particles, and is not described separately.

Considering the impact of surface roughness on the retrievals, $P_{34} / P_{11}$ is the only element for which the results deviate markedly from those for the original shapes. Cal and Dol exhibit a major effect, whereas the other particles are not affected noticeably. For both $\mathrm{Cal}$ and Dol, the refractive indices that produce the worst fits for the unroughened versions produce the best fits for the roughened version. The opposite is also true, especially for Cal: the refractive indices that produce good fits for the unroughened particle produce poor fits for the roughened particle.

\subsection{Fixed shape distribution}

In addition to allowing the ellipsoid shape distribution to vary while searching for the best-fitting $m$, we also investigate how the scattering error depends on $m$ when the shape distribution is fixed. For this, we use a uniform distribution that assigns equal weights for all shapes in the distribution, as per Eq. (11). The distribution therefore includes all 46 ellipsoidal shapes. Figure 4 shows the contour plots of $E_{i j}$ for this case.

The minimum scattering matrix element errors are found at the maximum real part and the minimum imaginary part of the refractive index for $P_{11}$ of the particle ensemble. In general, all refractive indices with the imaginary part less than 0.004 have relatively small errors regardless of the value of the real part. Similarly, all refractive indices with large imaginary parts have large errors. Cal, Dol and Agg are all very similar to the ensemble, while Sil is notably different. In fact, as was the case with the fitted shape distribution, the behavior of the error for Sil is opposite to that of the other particles. That is, Sil errors are small at all refractive indices with $m_{\mathrm{i}}=0.02$ and large at all small-to-medium imaginary refractive indices.

For the ensemble $P_{12} / P_{11}$, refractive indices with real parts larger than 1.45 and imaginary parts smaller than 0.01 produce good fits, with the error increasing only very mod- erately compared to that with $m_{\mathrm{opt}}$. Large imaginary refractive indices produce large errors, while small real refractive indices produce modest errors. Cal and Dol follow the behavior of the ensemble, while Agg and Sil differ from them but agree with each other. Both Agg and Sil have the minimum error at small real refractive indices and large imaginary refractive indices, which is in stark contrast to the other particles. Moreover, the refractive indices that produce small errors for the ensemble, Cal and Dol, produce large errors for Agg and Sil.

The $m_{\text {opt }}$ of $P_{22} / P_{11}$ for the ensemble lies at the maximum of the real axis and the minimum of the imaginary axis of the refractive index range used. The error increases smoothly as the refractive index goes further away from $m_{\text {opt }}$. High imaginary refractive indices produce large errors, whereas at low imaginary refractive indices almost every value of the real refractive index produces at most a modest error. Cal and Agg behave identically to the ensemble, but there are differences in the behaviors of Dol and Sil. Sil has the good-fit region of refractive indices at large real and small-to-medium imaginary refractive indices, like the ensemble, Cal and Dol, but the difference is that the refractive indices with the real part less than 1.5 produce large errors for Sil. Dol, however, has the optimum at minimum real and minimum imaginary refractive index. Large real and large imaginary parts of the refractive index are associated with large errors.

The $P_{33} / P_{11}$ contour map is very similar to the free shape distribution case for the ensemble, the main difference being that the error gradient is even smoother. Cal, Dol and Agg all resemble the ensemble very closely. Sil, however, differs from both the other particles as well as the behavior of Sil in the free shape distribution case. The optimum refractive index for Sil is located at the maximum real and maximum imaginary refractive index. Additionally, whereas most of the good-fit regions for the elements discussed so far have been convex, for Sil in this case the good-fit region is concave. 
Effectively, the behavior and the contour map of Sil are yet again inverse to that of the other particles and the ensemble.

For $P_{34} / P_{11}$, large real refractive indices produce small errors independently of the imaginary part. Conversely, small real refractive indices cause the errors to be large, almost regardless of the imaginary part. Cal, Agg and Sil resemble the ensemble, but Dol is clearly different. For Dol, the optimum refractive index is found at the minimum of the real and at the maximum of the imaginary part of the refractive index. Unlike the other elements, the fitting error for Dol is not independent of the imaginary part of the refractive index, and both small real parts and large imaginary parts produce large errors.

$P_{44} / P_{11}$ resembles $P_{33} / P_{11}$ very closely for the ensemble and all the particles and is therefore not described separately.

As in the case for the fitted shape distribution, $P_{34} / P_{11}$ is the only element that is affected by roughening to a significant degree. Interestingly, the roughened version of Dol resembles the non-roughened versions of the other particles quite closely and is therefore behaving almost oppositely to its own unroughened version. The behavior for $P_{34} / P_{11}$ of $\mathrm{Cal}$, which was greatly affected by roughening in the case that the shape distribution was fitted and not uniform, remains unaffected by roughening in the case of a uniform shape distribution.

\subsection{Synthesis}

From Figs. 3 and 4 and Tables 2 and 3 we see that in most cases $m_{\text {opt }}$ deviates significantly from $m_{\text {true }}$, the exact location depending on the element used for the retrieval. Furthermore, for several of the elements, $m_{\mathrm{opt}}$ varies greatly depending on the particle in question. Specifically, for almost all of the various particles and scattering matrix elements, $m_{\mathrm{opt}}$ is not close to $m_{\text {true }}$ regardless of whether the ellipsoid shape distribution is a free parameter or a fixed constant. Additionally, most of the $m_{\text {opt }}$ are at an extreme of either the real or the imaginary refractive index scales used in this study, which implies that even better fits might have been possible if the refractive index domain tested would have been wider. There are cases where $m_{\text {opt }}$ is near the $m_{\text {true }}$ value on either or both axes, but those seem to be exceptions. Moreover, the good-fit regions can be very large, which can make the retrieved refractive index extremely sensitive to small changes in the target particle features or measurement errors. This is exemplified in the text below. However, it is noteworthy that in several cases the good-fit region does include the real refractive index, and therefore it is not inconceivable that the retrieval process could result in the true refractive index in some cases.

When performing the analysis for particles with added artificial surface roughness, the results usually change only slightly (not shown). However, sometimes the results change dramatically, particularly for $P_{34}$. An example of a significant effect of roughening on the scattering error of the fitted shape distribution for $P_{34}$ of Dol is shown in Fig. 5. Although there are similarities in the unroughened and roughened versions of the error plots, there are also major differences. For example, $m_{\text {opt }}$ for the roughened version produces the worst fit of all of the refractive indices for the original version.

Due to the large variability in the optima location for different elements and particles, performing the retrieval using a combination of different elements can yield a wide variety of results, depending on the scaling and weighing of the error functions and individual scattering matrix elements. Most notably, in some cases almost any refractive index could be retrieved, were the weights or error definitions selected accordingly, because the individual $m_{\text {opt }}$ cover most of the extremes of the studied refractive index space. This means that any weighing must be done with great care. The flexibility of the combined element retrievals is illustrated in Fig. 6, where we use two different error scaling scenarios for a retrieval using both $P_{11}$ and $P_{12}$. In the first case, we simply use the sum of the error estimates of individual elements. This resembles the $P_{11}$ case very closely, because $P_{11}$ errors are larger and dominate the combined scattering error. In the second case, we scale the error ranges of each element to unity, and the retrieved refractive index changes significantly. The effect is even more pronounced if there are more than two scattering matrix elements involved due to greater flexibility in the individual optima locations. Although there is a definite theoretical interest in seeing the results based on all six scattering matrix elements, we chose not to include the six-element analysis in this work due to ambiguities related to the heavily varying individual optima locations, the limited refractive index range and the sensitivity of such analysis to scaling

Figure 7, finally, shows the target particle scattering matrix elements $P_{11}$ and $P_{12}$ together with the ellipsoid fits based on $m_{\text {opt }}$ and $m_{\text {true }}$. We see that the $m_{\text {opt }}$ fits are very good for the most part, which is yet another indication that ellipsoids really do form a very flexible base and are capable of producing good fits to the scattering matrix elements for various irregularly shaped scatterers. However, the $m_{\text {true }}$ fits are generally not nearly as good. The greater differences seen at large scattering angles of $P_{11}$ are features of the logarithmic $y$ axis; the absolute magnitudes of these errors are minuscule even though they look prominent in the figures. It should be noted that, had our refractive index ranges been more extensive, some of the fits would likely have been even better and the $m_{\text {opt }}$ even further away from $m_{\text {true }}$. However, it is evident that the true refractive index produces overall poor fits in many cases, and therefore the goodness of the fit is more of an evidence of the flexibility of the base and not of any inherent physical representativity of the fitted ellipsoid ensembles to the original particle.

It is important to consider various uncertainty sources for the retrieval. Because the discussion on the accuracy of the ellipsoid database is outside of the focus of this work, the main concerns are the DDA accuracy and the retrieval process reliability. DDA accuracy is discussed in detail in 


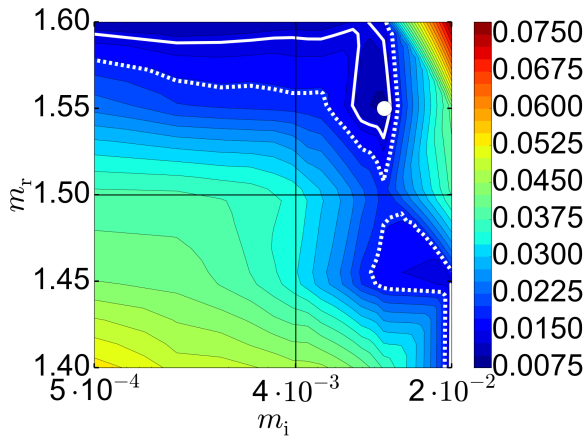

(a) Original unroughened version of Dol

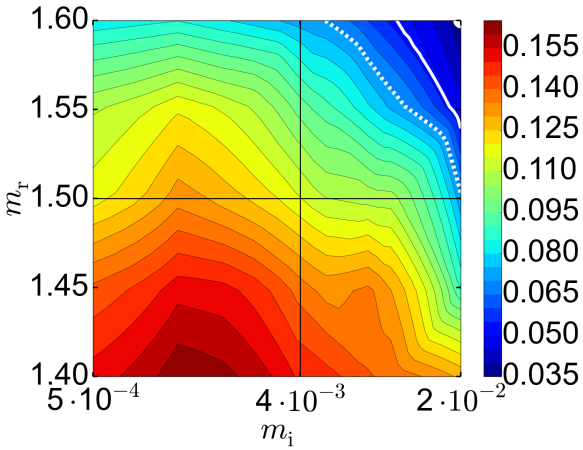

(b) Dol with surface roughening

Figure 5. Illustration of the effect of roughening on $P_{34}$ of Dol as in Fig. 3. The added modest surface roughness in (b) changes the behavior of the scattering error as function of the refractive index dramatically.

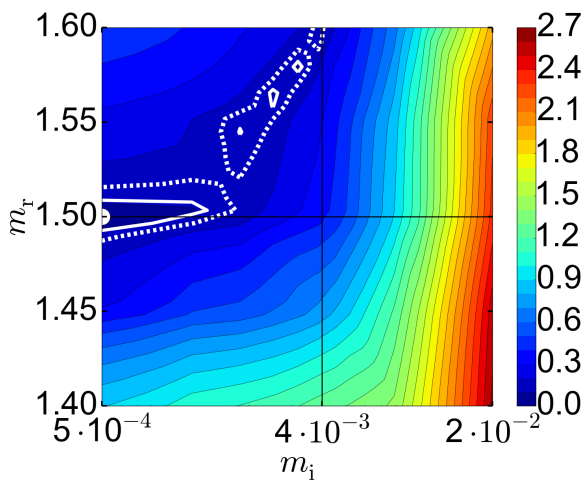

(a) No forced normalization

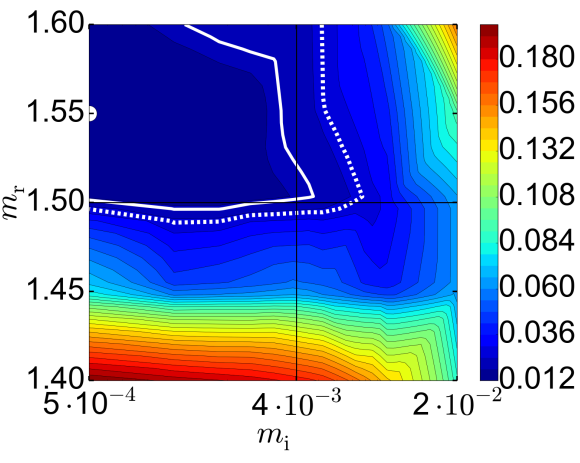

(b) Forced normalization

Figure 6. Illustration of two combined $P_{11}$ and $P_{12}$ normalization scenarios for the particle ensembles, as Fig. 3. (a) shows the sum the individual $E_{i j}$ directly such as they are, while (b) the individual errors are first scaled in such a way that the error range of each element is the same,: unity. The behavior of scattering error depends greatly on how the components of the multi-element errors are weighted.

Sect. 2.2 and seems sufficient for the conclusions of this paper. Retrieval process reliability was tested by performing the identical refractive index retrieval for a shape for which the correct refractive index should be found, that is, one of the shapes available for fitting. We chose to use spheroids (with an aspect ratio of 1.5) because of straightforward independent validation of scattering matrix data with T-matrix method, thus restricting one error source completely. The results of the retrieval test for the spheroid are not shown here, but for all scattering matrix element the retrieved refractive index was very close to the true refractive index or, in case of very large good-fit regions, had only a modest relative error. This slight mismatch is likely due to mild size-distribution averaging in the ellipsoid database and other similar minor discrepancies.

Based on these results, it seems that ellipsoids are not reliable for solving the inverse problem of retrieving the refractive index from scattering matrix data of irregular nonellipsoidal particles, especially when using only individual elements. The retrieval results may be good in some cases for specific combinations of elements but that appears to depend strongly on the details of the combination, and any combination that works in one case might not work in another. Small ensembles of ellipsoids, like those used here, do seem to provide good fits with the correct refractive index, but even better ones with erroneous refractive index. In fact, the good quality of the fits may actually give a misleading impression of the validity of ellipsoid fitting. However, it needs to be emphasized that the ensemble used in this study is a small one, based on just three particles, without any abundance-dependent weighing. Ensembles containing a larger number of different particles, such is the case in the atmosphere, might yield different results.

Clearly, one can not assume that matching optical parameters between two scatterers imply matching physical parameters. While this might be true for isolated cases, it does not hold in the general case. This also opens up questions about solving the inverse problem. Even if a set of model particles is able to replicate some optical data with freely adjustable weights, thus formally solving the forward problem for each 

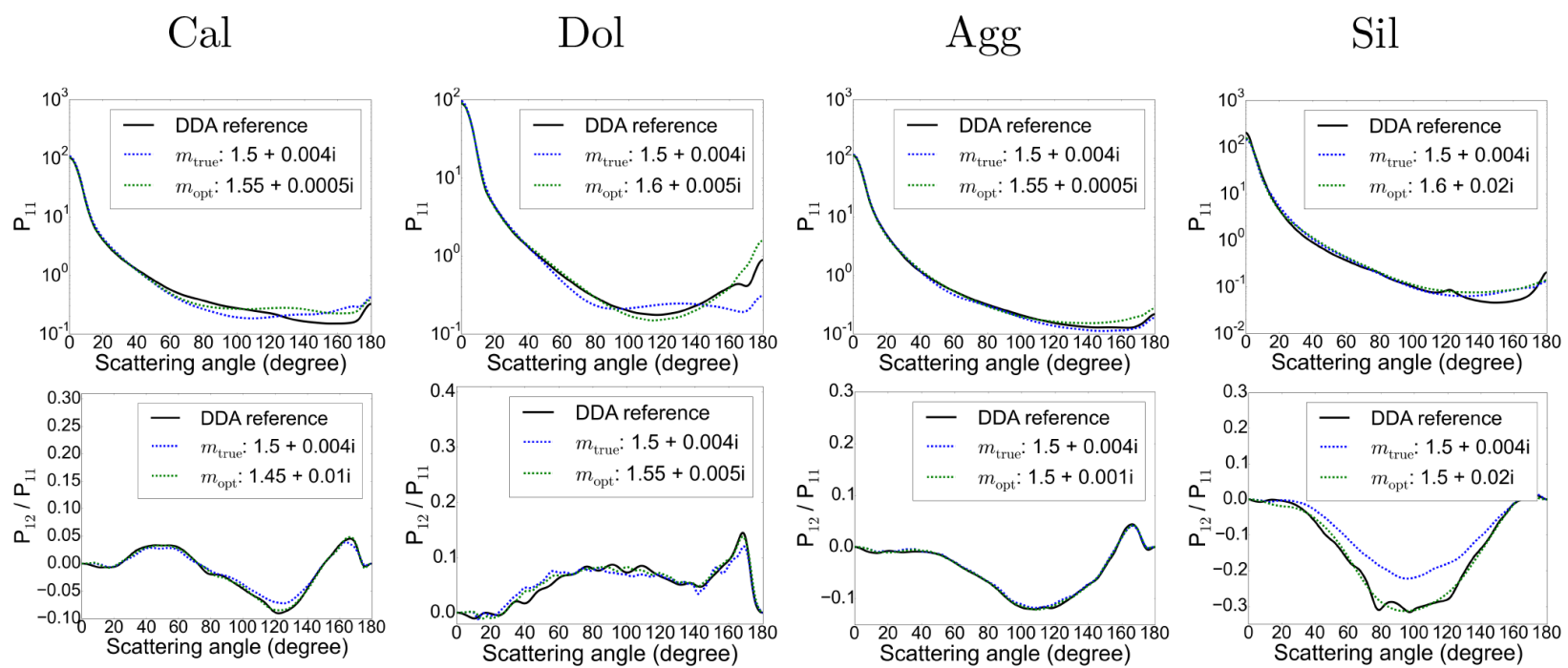

Figure 7. Target scattering matrix elements for $P_{11}$ and $P_{12} / P_{11}$ for the four individual particles as well as the best fits produced by the true refractive index $\left(m_{\text {true }}\right)$ and the optimum refractive index $\left(m_{\text {opt }}\right)$ ellipsoid bases.

parameter individually, can we trust that the physical parameters are also close? This would be a requirement for said model particles to be used in retrieval, but based on this study, it seems like this assumption does not hold, especially if the model shapes do not even closely match the target shape.

\subsection{Implications for radiative transfer}

In addition to seeing the retrieval errors of refractive indices, it is interesting to know how the inaccurate retrievals of refractive index propagate into higher-level applications, such as radiative transfer simulations. To investigate this, we calculated the single-scattering albedo $(\omega)$ and the asymmetry parameter $(g)$ for the fitted ellipsoid shape distributions of $m_{\text {opt }}$ retrieved via each of the individual scattering matrix elements. The results, as well as the true parameter values calculated from the DDA particle ensemble, called REF in the text and figures below, are shown in Fig. 8. We see that apart from the retrieval based on $P_{34} / P_{11}$, the retrieved parameter values are reasonably well clumped together, especially with regards to the single-scattering albedo. However, this clumping can be misleading, since the REF values of the parameters are outside of the group on both axes: both the REF single-scattering albedo and the target asymmetry parameter are lower than those retrieved based on any of the scattering matrix elements except for $P_{34} / P_{11}$. The retrieval based on $P_{34} / P_{11}$ is a clear outlier and is farther away from the target case on both axes than any of the others.

We next consider the aerosol radiative effects on the topof-the-atmosphere (TOA) and surface (SFC) net fluxes and atmospheric absorption (ATM), normalized by the down-

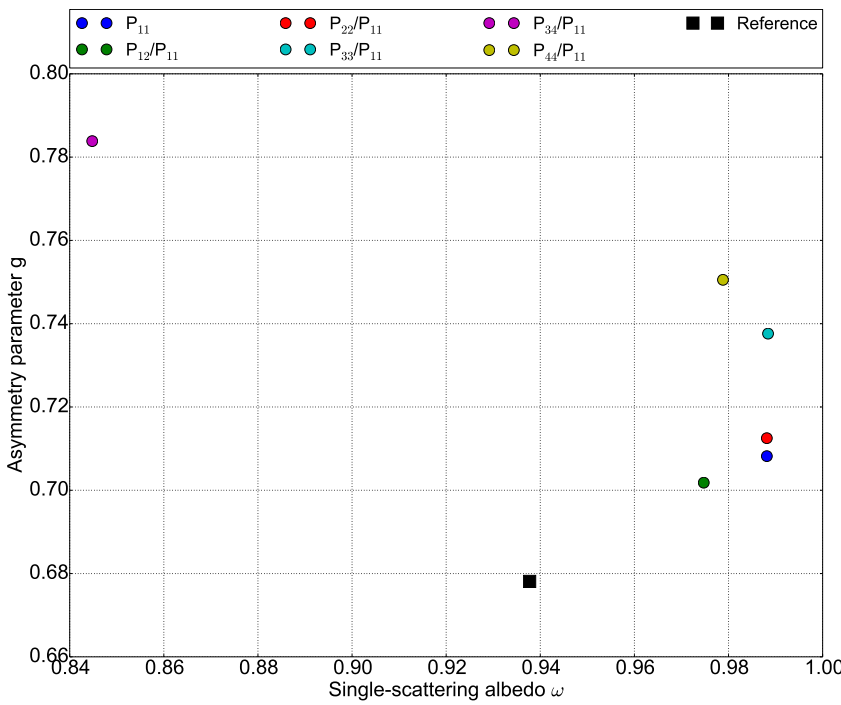

Figure 8. Variability of single-scattering albedo and asymmetry parameter when the retrieval is based on fitting the ellipsoid shape distribution to individual scattering matrix elements of the particle ensemble. The true values of these parameters for the particle ensemble are also shown for reference.

welling solar flux $F_{\mathrm{TOA}}^{\downarrow}$ at TOA:

$$
\begin{aligned}
f_{\mathrm{TOA}} & =\frac{F_{\mathrm{TOA}}^{\text {net }}(\text { aer })-F_{\mathrm{TOA}}^{\text {net }}(\text { no aer })}{F_{\mathrm{TOA}}^{\downarrow}}, \\
f_{\mathrm{SFC}} & =\frac{F_{\mathrm{SFC}}^{\text {net }}(\text { aer })-F_{\mathrm{SFC}}^{\text {net }}(\text { no aer })}{F_{\mathrm{TOA}}^{\downarrow}}, \\
f_{\mathrm{ATM}} & =f_{\mathrm{TOA}}-f_{\mathrm{SFC}} .
\end{aligned}
$$

Here $F_{\text {net }}$ refers to the net (down - up) radiative flux either in the presence (aer) or absence (no aer) of the aerosol layer. 
Two cases are contrasted: the REF case, for which $\omega=$ 0.9377 and $g=0.6781$, and the retrieval based on P11 (hereafter, the P11-fit) for which $\omega=0.9881$ and $g=0.7082$. Computations were done for the wavelength of $0.55 \mu \mathrm{m}$, for mineral aerosol optical depths (AODs) ranging from 0.05 (background conditions) to 3 (a strong dust storm). While the misrepresentation of aerosol shape might, in reality, also influence the AOD retrievals, it is assumed here that the AOD is identical for the REF and $P_{11}$-fit cases, so that the differences between them arise from $\omega$ and $g$ only. Following Haapanala et al. (2012), the aerosol was placed in the lowest $3 \mathrm{~km}$ of a cloud-free tropical model atmosphere with water vapor reduced by $50 \%$ compared to the standard tropical profile of Anderson et al. (1986). Two values were considered for the surface albedo, $\alpha_{\mathrm{s}}=0.07$ and $\alpha_{\mathrm{s}}=0.3$. Optical depths for gaseous absorption and Rayleigh scattering were computed using the scheme of Freidenreich and Ramaswamy (1999), and multiple scattering was handled using DISORT (Stamnes et al., 1988), with eight streams and $\delta$-M scaling included. Henyey-Greenstein phase function (Henyey and Greenstein, 1941) was assumed for the aerosols.

Figure $9 \mathrm{a}-\mathrm{c}$ show $f_{\mathrm{TOA}}, f_{\mathrm{SFC}}$ and $f_{\mathrm{ATM}}$ as a function of AOD, and Fig. 9d-f show the corresponding fractional differences between the $P_{11}$-fit and REF cases. The cosine of solar zenith angle is fixed at $\mu_{0}=0.6$, but the main features were similar for other solar elevations as well. The following points can be made:

1. Due to the larger $\omega$ for the $P_{11}$-fit case, aerosol absorption is reduced dramatically, so that $f_{\mathrm{ATM}}$ is $71-76 \%$ smaller than in the REF case (Fig. 9c and f).

2. The larger $\omega$ (i.e., reduced absorption) and larger $g$ (i.e., reduced backward scattering) in the $P_{11}$-fit case both make the aerosol layer more transmissive (Fig. 9b and e). Consequently, $f_{\mathrm{SFC}}$ is $26-30 \%(31-37 \%)$ smaller than in the REF case for $\alpha_{\mathrm{s}}=0.07\left(\alpha_{\mathrm{s}}=0.30\right)$.

3. For $f_{\mathrm{TOA}}$, the effects of larger $\omega$ and larger $g$ in the $P_{11}$-fit case are compensatory. However, the former factor dominates, which results in a larger negative $f_{\text {TOA }}$ (Fig. 9a and d). For a low surface albedo $\alpha_{\mathrm{s}}=0.07$, the difference to REF is small for low AOD (e.g., $\approx 5 \%$ for $\mathrm{AOD}=0.1$ ) but it increases to nearly $40 \%$ for $\mathrm{AOD}=$ 3 . For a high surface albedo $\alpha_{\mathrm{s}}=0.30$, the differences are larger both in an absolute sense and (especially) in a relative sense, 120-200\%.

Overall, this example suggests that errors in refractive index arising from inaccurate shape assumptions in the retrieval scheme may result in very substantial errors in the single-scattering parameters (especially $\omega$ ) and in the resulting aerosol radiative effects. The detailed results are, of course, sensitive to the actual retrieval algorithm used.

\section{Summary and conclusions}

In this work we investigated the reliability of the ellipsoid ensemble fitting for retrieving refractive indices of non-ellipsoidal model particles, with shapes retrieved from real dust particles via stereogrammetry. While it is known that ellipsoid ensembles can replicate the scattering of nonellipsoidal particles closely, it is not known whether such ensembles are linked to the microphysical properties of the target particles. That is, if an ellipsoid shape ensemble of a given refractive index fit the scattering data of a particle extremely closely, does it guarantee that the particle has the same refractive index? This is the implicit assumption that is made in various retrieval processes, but the validity has not been investigated thoroughly before.

This question was studied with a two-step process. First we performed fitting of the scattering matrix elements of ellipsoid ensembles of various refractive indices. Second, we investigated the relationships of the scattering errors of the best-fit ensembles and the deviation of the refractive index of this best-fit ensemble from the true refractive index of the target particle, which was known. As target particles we used individual stereogrammetric particles as well as a small ensemble of them. In addition to having the ellipsoid shape distribution as a free parameter, we investigated the scattering matrix element differences between the target particles and a uniform distribution of ellipsoid shapes.

Based on our results, ellipsoid fitting is not a reliable method for retrieving the true refractive index of nonellipsoidal irregular particles, despite producing good fits to the scattering matrix elements. The retrieval based on error minimization found the true refractive index for only three cases out of 120 shown in Tables 2 and 3. In fact, the scattering error of the ellipsoids with the correct refractive index can be significantly higher than that of a wrong refractive index. This implies fundamental problems in modeling scattering properties of irregular particles by simplified model particles. Importantly, the ellipsoid ensemble with the real refractive index does produce good fits in several cases, even when ensembles with wrong refractive indices produce even better fits. Depending on which scattering matrix elements are used, the real and the imaginary part may be either smaller or larger compared to the true refractive index, and therefore the retrieved refractive index can not be used even to restrict the estimate of the true refractive index from any direction. The retrieved refractive index acquired by using several matrix elements at once can be better than those of any individual elements, but that seems to be a case of several wrong solutions averaging to a decent one by pure chance. However, the validity of multi-element fitting can not be investigated reliably at this time due to the limited range of refractive indices in the ellipsoid database that does not cover all of the individual scattering matrix element optima.

When using the modified model particles with added surface roughness, the retrieval results are usually not af- 

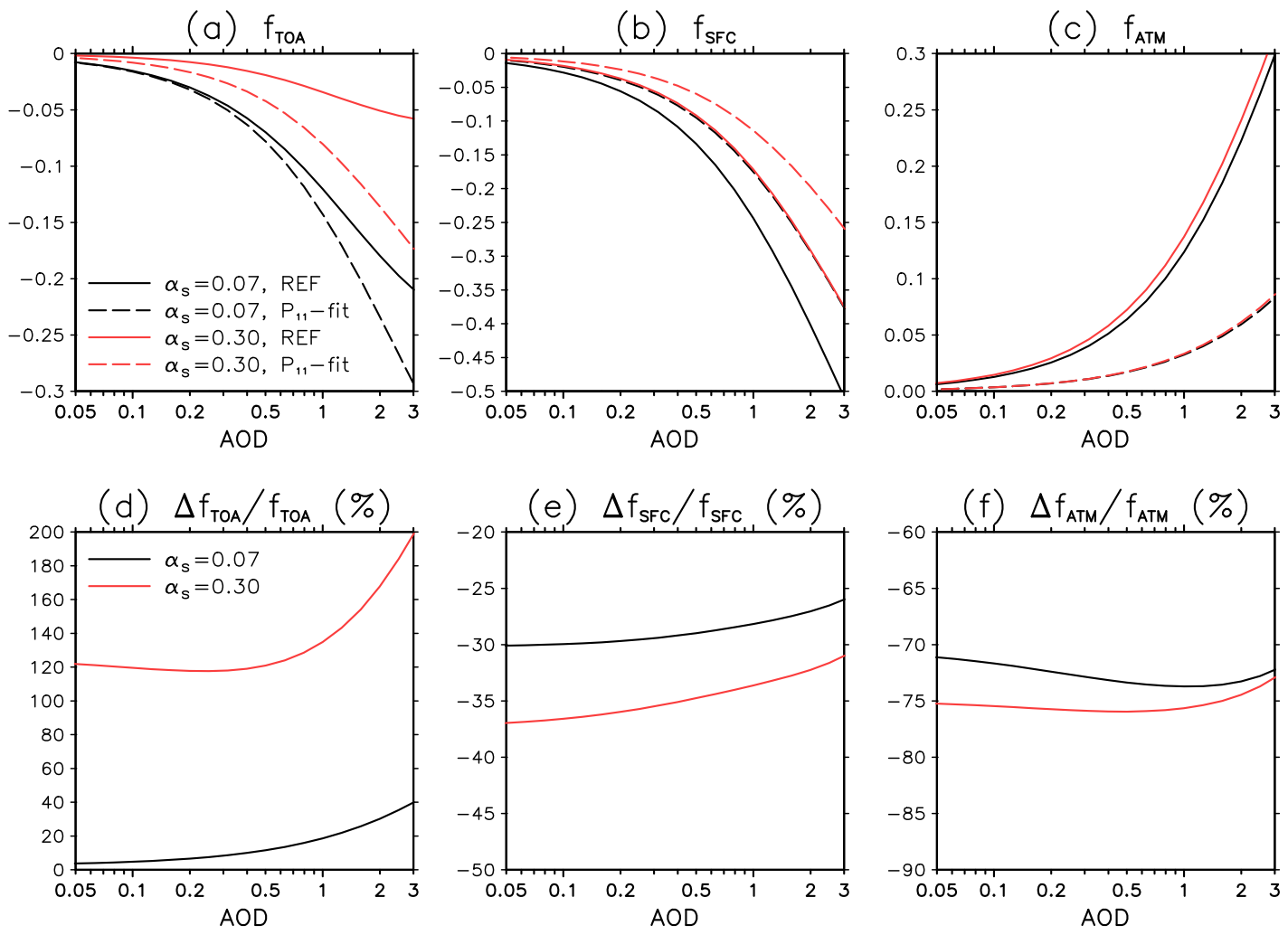

Figure 9. (a-c) Normalized aerosol radiative effects (Eqs. 12-14) for the REF (solid lines) and $P_{11}$-fit cases (dashed lines) for surface albedos of $\alpha_{\mathrm{S}}=0.07$ (black) and $\alpha_{\mathrm{S}}=0.30$ (red). (d-f) Corresponding relative differences (in \%) between the $P_{11}$-fit and REF cases.

fected much. Incidentally, for most scattering matrix elements the scattering errors increase, suggesting that ellipsoids do a poorer job at mimicking scattering by dust particles with added surface roughness. The retrieval of $m$ works as poorly as for the original target particles. In some cases, though, the effect can be dramatic, such as the $m_{\mathrm{opt}}$ moving from one extreme of either of the refractive index axes to the other extreme. This indicates further difficulties in retrieving the refractive index of rough particles using smooth model particles.

Overall, it seems that the refractive index ranges selected were not completely sufficient to find the actual best-fit values, because most retrieved refractive indices were on edge of our complex refractive index space. However, the purpose of this study was not to find the refractive indices with the absolutely best match but rather to investigate whether the refractive index can be retrieved from the angular dependence of scattering from irregular dust particles using simplified model particles.

The analysis results clearly show that the retrieval of $m$ fails, regardless of whether the ellipsoidal shape distribution is fixed or allowed to vary. Further, the retrieved refractive indices depend on which element or element combinations are used, implying inconsistencies in the performance of ellipsoids. It thus seems that ellipsoids are ill-suited for refractive index retrieval of irregularly shaped non-ellipsoidal particles from light scattering data. Importantly, it was demonstrated that the resulting errors in single-scattering albedo and asymmetry parameter have the potential to produce major errors in computing the aerosol radiative effects.

When considering the practical implications of our findings, we must emphasize that few actual retrieval methods are based on an approach adapted here. Additionally, many applications use either spheroids or spheres instead of ellipsoids and have different limitations and error sources than those of ellipsoids. Different instruments employ different types of measurement data, for example, and thus have different vulnerabilities to the inherent biases imposed by the ellipsoid model. Also, we only considered cases with individual particles or a very small ensemble of three particles. Additionally, our target particles may not scatter light like real dust particles even though their shapes are directly derived from those of real dust particles. Therefore, this study should not be taken as a proof that dust refractive index retrieval using ellipsoids does not work. Rather, this study should be considered a cautionary tale that hopefully encourages retrieval teams to test their algorithm with sufficiently realistic reference data, yet we need to emphasize that our retrieval tests were conducted under ideal conditions. We did not have any measurement errors, other external contributions to the 
"measured" radiation, and we automatically employed the correct size distribution. We note that size and refractive index often have similar effects on scattering, so retrieval of both the size and refractive index may give rise to even larger retrieval errors due to error compensation.

Based on our findings, it would be interesting to carry out similar investigation employing more complex model shapes for the retrieval. Unfortunately, the computational burden of such an investigation would be tremendous. One possible method to facilitate such a study is the shape matrix method by Petrov et al. (2006), which allows relatively fast computations for different refractive indices and sizes, once the shape-dependent shape matrix has been solved. Another suitable recent development is the invariant imbedding T-matrix method, which allows for fast optical calculations of various scatterers, such as ice crystals and dust particles (Bi and Yang, 2014). Additionally, it would be tremendously helpful to be able to predict scattering errors from model shape differences. While it seems like a very hard thing to do, there have been semi-empirical approaches by Strokotov et al. (2009) and Moskalensky et al. (2013) that have experimented with the idea. While their approach is not directly applicably for dust particle shapes, it is very encouraging to see progress in this area.

Acknowledgements. The authors wish to thank Lei Bi, Maxim Yurkin, David Crisp and an anonymous referee for their helpful comments in improving the manuscript. This research has been funded, in part, by the Academy of Finland (grant 255718) and the Finnish Funding Agency for Technology and Innovation (Tekes; grant 3155/31/2009). Maxim Yurkin is acknowledged for making his ADDA code publicly available (https://code.google.com/p/a-dda/). Additionally, we are grateful to Hannakaisa Lindqvist and Zhaokai Meng for providing the stereogrammetric shapes and the ellipsoid database, respectively. Both the ellipsoid and the DDA data are available on request via the lead author (osku.kemppinen@fmi.fi).

Edited by: P. Formenti

\section{References}

Anderson, G., Clough, S., Kneizys, F., Chetwynd, J., and Shettle, E.: AFGL Atmospheric Constituent Profiles (0-120 km), Tech. Rep. AFGL-TR 86-0110, Air Force Geophys. Lab., Hanscom Air Force Base, Bedford, Mass., 1986.

Bi, L. and Yang, P.: Accurate simulation of the optical properties of atmospheric ice crystals with the invariant imbedding T-matrix method, J. Quant. Spectrosc. Ra., 138, 17-35, 2014.

Bi, L., Yang, P., Kattawar, G., and Kahn, R.: Single-scattering properties of triaxial ellipsoidal particles for a size parameter range from the Rayleigh to geometric-optics regimes, Appl. Optics, 48, 114-126, 2009.

Draine, B. T. and Flatau, P. J.: Discrete-dipole approximation for scattering calculations, J. Opt. Soc. Am. A, 11, 1491-1499, 1994.
Dubovik, O., Sinyak, A., Lapyonok, T., Holben, B. N., Mishchenko, M., Yang, P., Eck, T. F., Volten, H., Muñoz, O., Veihelmann, B., van der Zande, W. J., Leon, J.-F., Sorokin, M., and Slutsker, I.: Application of spheroid models to account for aerosol particle nonsphericity in remote sensing of desert dust, J. Geophys. Res., 111, D11208, doi:10.1029/2005JD006619, 2006.

Durant, A., Harrison, S., Watson, I., and Balkanski, Y.: Sensitivity of direct radiative forcing by mineral dust to particle characteristics, Prog. Phys. Geog., 33, 80-102, 2009.

Freidenreich, S. M. and Ramaswamy, V.: A new multiple-band solar radiative parameterization for general circulation models, J. Geophys. Res., 104, 31389-31409, doi:10.1029/1999JD900456, 1999.

Haapanala, P., Räisänen, P., Kahnert, M., and Nousiainen, T.: Sensitivity of the shortwave radiative effect of dust on particle shape: comparison of spheres and spheroids, J. Geophys. Res., 117, D08201, doi:10.1029/2011JD017216, 2012.

Haywood, J. M., Johnson, B. T., Osborne, S. R., Mulcahy, J., Brooks, M. E., Harrison, M. A. J., Milton, S. F., and Brindleye, H. E.: Observations and modelling of the solar and terrestrial radiative effects of Saharan dust: a radiative closure case-study over oceans during the GERBILS campaign, Q. J. Roy. Meteor. Soc., 137, 1211-1226, doi:10.1002/qj.770, 2011.

Henyey, L. G. and Greenstein, J. L.: Diffuse radiation in the galaxy, Astrophys. J., 93, 70-83, 1941.

Hovenier, J. and van der Mee, C.: Basic relationships for matrices describing scattering by small particles, in: Light Scattering by Nonspherical Particles, edited by: Mishchenko, M. I., Hovenier, J. W., and Travis, L. D., Academic Press, San Diego, Chapter 3, 61-85, 2000.

Kahnert, F. M., Stamnes, J. J., and Stamnes, K.: Using simple particle shapes to model the Stokes scattering matrix of ensembles of wavelength-sized particles with complex shapes: possibilities and limitations, J. Quant. Spectrosc. Ra., 74, 167-182, 2002.

Kemppinen, O., Nousiainen, T., and Lindqvist, H.: The impact of surface roughness on scattering by realistically shaped wavelength-scale dust particles, J. Quant. Spectrosc. Ra., 150, 55-67, doi:10.1016/j.jqsrt.2014.05.024, 2015.

Lindqvist, H., Jokinen, O., Kandler, K., Scheuvens, D., and Nousiainen, T.: Single scattering by realistic, inhomogeneous mineral dust particles with stereogrammetric shapes, Atmos. Chem. Phys., 14, 143-157, doi:10.5194/acp-14-143-2014, 2014.

Meng, Z., Yang, P., Kattawar, G., Bi, L., Liou, K., and Laszlo, I.: Single-scattering properties of tri-axial ellipsoidal mineral dust aerosols: A database for application to radiative transfer calculations, J. Aerosol Sci., 41, 501-512, doi:10.1016/j.jaerosci.2010.02.008, 2010.

Merikallio, S., Lindqvist, H., Nousiainen, T., and Kahnert, M.: Modelling light scattering by mineral dust using spheroids: assessment of applicability, Atmos. Chem. Phys., 11, 5347-5363, doi:10.5194/acp-11-5347-2011, 2011.

Merikallio, S., Nousiainen, T., Kahnert, M., and Harri, A.M.: Light scattering by the Martian dust analog, palagonite, modeled with ellipsoids, Opt. Express, 21, 17972-17985, doi:10.1364/OE.21.017972, 2013.

Mishchenko, M. I. and Travis, L. D.: Capabilities and limitations of a current Fortran implementation of the $T$-matrix method for randomly oriented rotationally symmetric scatterers, J. Quant. Spectrosc. Ra., 60, 309-324, 1998. 
Mishchenko, M. I., Travis, L. D., Kahn, R. A., and West, R. A.: Modeling phase functions for dustlike tropospheric aerosols using a shape mixture of randomly oriented polydisperse spheroids, J. Geophys. Res., 102, 16831-16847, 1997.

Moskalensky, A. E., Yurkin, M. A., Konokhova, A. I., Strokotov, D. I., Nekrasov, V. M., Chernyshev, A. V., Tsvetovskaya, G. A., Chikova, E. D., and Maltsev, V. P.: Accurate measurement of volume and shape of resting and activated blood platelets from light scattering, J. Biomed. Optics, 18, 017001-017001, 2013.

Muñoz, O., Moreno, F., Guirado, D., Ramos, L., López, A., Girela, F., Jerónimo, J., Costillo, L., and Bustamante, I.: Experimental determination of scattering matrices of dust particles at visible wavelengths: The IAA light scattering apparatus, J. Quant. Spectrosc. Ra., 111, 187-196, 2010.

Nousiainen, T. and Kandler, K.: Light scattering by atmospheric mineral dust particles, in: Light Scattering Reviews 9, edited by: Kokhanovsky, A. A., Springer Praxis Books, Springer, Berlin, Heidelberg, 3-52, doi:10.1007/978-3-642-37985-7, 2015.

Nousiainen, T., Zubko, E., Niemi, J. V., Kupiainen, K., Lehtinen, M., Muinonen, K., and Videen, G.: Single-scattering modeling of thin, birefringent mineral dust flakes using the discrete-dipole approximation, J. Geophys. Res., 114, D07207, doi:10.1029/2008JD011564, 2009.

Nousiainen, T., Kahnert, M., and Lindqvist, H.: Can particle shape information be retrieved from light-scattering observations using spheroidal model particles?, J. Quant. Spectrosc. Ra., 112, 22132225, doi:10.1016/j.jqsrt.2011.05.008, 2011.

Nousiainen, T., Zubko, E., Lindqvist, H., Kahnert, M., and Tyynelä, J.: Comparison of scattering by different nonspherical, wavelength-scale particles, J. Quant. Spectrosc. Ra., 113, 23912405, doi:10.1016/j.jqsrt.2012.03.032, 2012.

Penttilä, A., Zubko, E., Lumme, K., Muinonen, K., Yurkin, M., Draine, B., Rahola, J., Hoekstra, A., and Shkuratov, Y.: Comparison between discrete dipole implementations and exact techniques, J. Quant. Spectrosc. Ra., 106, 417-436, 2007.
Petrov, D., Synelnyk, E., Shkuratov, Y., and Videen, G.: The Tmatrix technique for calculations of scattering properties of ensembles of randomly oriented particles with different size, J. Quant. Spectrosc. Ra., 102, 85-110, 2006.

Press, W. H., Teukolsky, S. A., Vetterling, W. T., and Flannery, B. P.: Numerical Recipes in FORTRAN, 2nd Edn., Cambridge University Press, UK, 1992.

Purcell, E. M. and Pennypacker, C. R.: Scattering and absorption of light by nonspherical dielectric grains, Astrophys. J., 186, 705$714,1973$.

Stamnes, K., Tsay, S.-C., Wiscombe, W., and Jayaweera, K.: Numerically stable algorithm for discrete-ordinate-method radiative transfer in multiple scattering and emitting layered media, Appl. Optics, 27, 2502-2509, 1988.

Strokotov, D. I., Yurkin, M. A., Gilev, K. V., Van Bockstaele, D. R., Hoekstra, A. G., Rubtsov, N. B., and Maltsev, V. P.: Is there a difference between T-and B-lymphocyte morphology?, J. Biomed. Opt., 14, 064036-064036, 2009.

Waterman, P. C.: Matrix formulation of electromagnetic scattering, Proc. IEEE, 53, 805-812, 1965.

Yurkin, M. A. and Hoekstra, A. G.: The discrete-dipoleapproximation code ADDA: capabilities and known limitations, J. Quant. Spectrosc. Ra., 112, 2234-2247, 2011.

Zender, C. S., Bian, H., and Newman, D.: The mineral Dust Entrainment And Deposition (DEAD) model: description and 1990s dust climatology, J. Geophys. Res., 108, D14, doi:10.1029/2002JD002775, 2003.

Zubko, E., Petrov, D., Grynko, Y., Shkuratov, Y., Okamoto, H., Muinonen, K., Nousiainen, T., Kimura, H., Yamamoto, T., and Videen, G.: Validity criteria of the discrete dipole approximation, Appl. Optics, 49, 1267-1279, 2010.

Zubko, E., Muinonen, K., Muñoz, O., Nousiainen, T., Shkuratov, Y., Sun, W., and Videen, G.: Light scattering by feldspar particles: comparison of model agglomerate debris particles with laboratory samples, J. Quant. Spectrosc. Ra., 131, 175-187, 2013. 